\title{
EDITORIAL
}

\section{Ethnic variations in acute coronary syndromes}

\section{G A Lanza}

Heart 2004;90:595-597. doi: 10.1136/hrt.2003.026476

\begin{abstract}
Although it is very likely that ethnic variations in the incidence and, possibly, clinical outcome of acute coronary artery disease events exist, the causes for such differences are many and difficult to address fully, given the complex interplay of contributing factors
\end{abstract}

$\mathrm{P}$ opulation registries and epidemiological surveys have shown relevant differences in the incidence of clinical events caused by atherosclerotic coronary artery disease (CAD) among different countries in the world. Overall, these studies highlighted that $\mathrm{CAD}$ event rates are higher in North Europe and North America, and lower in Mediterranean and Oriental populations (for example, Chinese and Japanese). ${ }^{12}$ The obvious issue raised by these observations is whether differences are merely connected to environmental, nutritional and lifestyle factors, or whether genetic factors play any relevant role.

The importance of acquired factors is well known. Risk factors for CAD have been clearly identified in economically developed western countries and they are almost absent in underdeveloped societies, where CAD event rates are low. Accordingly, in developing areas the acquisition of western lifestyles is associated with a significant increase in CAD events, a phenomenon also observed in people who have emigrated from underdeveloped towards industrialised countries. ${ }^{3}$ In contrast, whether and in which way genetic factors influence ethnic differences in CAD events has not adequately been investigated yet.

Multiracial societies, including people belonging to different ethnic groups, exposed to similar environmental factors, may provide the unique possibility to explore the role of genetic factors in CAD events. These studies, however, may be difficult to interpret as minority ethnic groups often maintain most of their own habits for several generations and, compared to the predominant population, usually have lower social, educational, and economic conditions, thus making it difficult to discriminate between acquired and possible genetic determinants of risk.

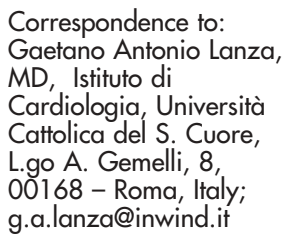

\section{ETHNIC VARIATIONS IN CAD EVENTS}

In this issue, Mak and colleagues ${ }^{4}$ report data on sex differences in coronary mortality following an acute myocardial infarction (AMI) among the three major ethnic groups which form the population of the city-state of Singapore-that is, Chinese (61.8\%), Indians ( $17.5 \%)$, and Malays
$(19.0 \%)$. Data are derived from the Singapore Myocardial Infarction Register (SMIR), which enrolled all potential cases of AMI in 25-64 year old people living in Singapore from 1991 to 1999. This report is an extension of the recently published data about AMI incidence and mortality in this population. ${ }^{5}$

The first major finding emerging from the SMIR database is a higher incidence of AMI in Indians and in Malays compared to Chinese (age standardised odds ratio 3.1 and 2.1 , respectively). ${ }^{5}$ While other data on Malays are lacking, the higher risk of AMI in Indians is consistent with that observed in other countries, ${ }^{67}$ and the lower risk in Chinese with that observed in previous epidemiological reports. ${ }^{12}$

These results may suggest that some genetic factors can make Indians predisposed to, and/or Chinese protected against, acute CAD events. Previous studies have indeed shown that Indians present a peculiar cardiovascular risk profile, characterised by a high prevalence of insulin resistance, glucose intolerance, central obesity, and diabetes, together with increased blood concentrations of other CAD risk indicators, including the plasminogen activator inhibitor-1, homocysteine, and lipoprotein(a). ${ }^{6}$ However, there is no definite evidence that this risk pattern is related to genetically determined ethnic factors and that it is directly responsible for the higher risk of CAD events in this ethnic group.

\section{Acquired factors}

Acquired factors have, in fact, been suggested to contribute significantly to the increased risk in Indians; these include a high consumption of clarified fat (ghee), resulting in dyslipidaemia, and a reduced probability to receive timely interventions for CAD prevention and care, due to a combination of cultural and socioeconomic factors, but also due to a frequent atypical CAD presentation, which may delay involvement of the healthcare system. ${ }^{6}$ Furthermore, a recent study failed to show an increased prevalence of factor $\mathrm{V}$ (Leiden) and thrombomodulin gene polymorphisms, potentially related to thrombogenesis, in Indians. ${ }^{8}$

Similar issues are found when assessing ethnic differences in CAD events in other geographic contexts. In the USA, the few studies comparing CAD events in black and white people provided complex and even contrasting results. Taken together, data seem to suggest that black people, compared to whites, may present similar or
Abbreviations: ACS, acute coronary syndromes; $A M I$, acute myocardial infarction; $C A D$, coronary artery disease; SMIR, Singapore Myocardial Infarction Register 
reduced risk but they also suggest that further ethnic differences might exist among specific age/sex subgroups. ${ }^{9}{ }^{10}$

\section{Age and sex differences}

In the National Health and Nutrition Examination Survey (NHANES) I epidemiologic follow up study, ${ }^{10}$ involving 11406 subjects, the risk adjusted hazard of CAD events was lower in both black men and women, compared to white subjects (relative risk (RR) 0.69 and 0.87 , respectively). Yet black young women (25-54 years old) showed a higher age adjusted risk for coronary death (RR 2.25), compared to white young women, although the difference became nonsignificant after correction for risk factors (RR 1.17, 95\% confidence limits (CL) 0.58 to 2.37). On the other hand, the risk for CAD events was higher in post-menopausal black women, compared to white women, enrolled in the heart and estrogen/progestin replacement study (RR 2.05), although the difference was reduced after adjustment for confounding factors (RR 1.52, 95\% CL 1.04 to 2.21 ). ${ }^{10}$

The reasons for this complex picture are not clear. Black people usually have higher rates of risk factors, including hypertension and diabetes, yet they usually show less severe CAD at angiography ${ }^{10}$ and even lower degrees of coronary calcification on computed tomography. Furthermore, black people have lower socioeconomic status and education, which have also been associated with increased risk. Thus, the similar or lower prevalence of CAD events in blacks may suggest either some genetic protective factors or maintenance of some unspecified healthier protective habits. The worse risk factor and social profile, combined with the low CAD incidence, however, might be penalising for black women, resulting in selection bias of the worst cases and delay in clinical CAD diagnosis and treatment.

Independent of the causes, ethnic variations in the incidence of CAD events may be associated, at least in some cases, with certain differences in the pathogenesis of acute coronary syndromes (ACS). Thus, in a recent study Japanese patients with AMI were found to have a significantly higher frequency of inducible vasospasm, both in the infarct and non-infarct related artery $(67 \%$ and $39 \%$, respectively), compared to Italian patients (23\% and $11 \%$, respectively), suggesting that coronary spasm might be involved more frequently in causing ACS in Japanese than in western populations. ${ }^{12}$

\section{ETHNIC VARIATIONS IN FATALITY RATES FOLLOWING ACUTE CORONARY SYNDROMES}

A second intriguing issue raised by the SMIR database is the possibility that mortality associated with AMI also might differ among ethnic groups, not necessarily paralleling AMI incidence, and that the differences might mainly concern specific age/sex subgroups, rather than the ethnic groups as a whole. In Mak's study, ${ }^{4}$ the 28 day fatality rates were indeed higher in Malays, compared to the two other ethnic groups. Sex analysis, however, revealed that whereas fatality rates differed significantly among women, with persistence of the differences at long term follow up, there were no significant differences among men. ${ }^{4}$

The higher mortality in Malay women does not seem to be explained by differences in clinical characteristics. Furthermore, the differences among groups were substantially related to pre-hospital fatality rates, thus excluding a major role for variations in acute hospital care or response to treatment (for example, thrombolysis, revascularisation procedures). Thus, although more extensive CAD and increased fatal arrhythmias cannot be excluded, it is possible that the higher death rates in the minority ethnic group of Malay women might again be due, at least in part, to a selection bias of most severe patients, or to a delay in diagnosis and recurrence to medical care.

The suggestion that AMI related mortality may differ among ethnic groups has previously received limited attention. Investigations in the USA have provided contrasting data, with studies showing lower, higher or no differences between black and non-black, patients ${ }^{13}{ }^{14}$; these studies, however, are limited by the inclusion of in-hospital deaths only. Furthermore, differences between ethnic groups in baseline clinical characteristics, and sometimes also in acute management, may have influenced clinical outcome. Overall, whether a different ethnic susceptibility to death following ACS does exist is an intriguing but unsettled question.

Regardless of ethnic considerations, Mak's data, on the other hand, lend support to the hypothesis of increased AMI related fatality rates in women, as compared to men. ${ }^{4}$ The higher mortality in women reported in previous studies was largely attributed to worse clinical features at presentation, ${ }^{215}$ and it has also been suggested that it might actually be apparent, concerning only in-hospital deaths, which would be counterbalanced by a higher pre-hospital mortality in men. ${ }^{15}{ }^{16}$ In the SMIR registry, however, both pre- and inhospital adjusted fatality rates were consistently higher in women in all ethnic groups. Thus, taken together, these and previous data ${ }^{215}$ may suggest that sex differences in AMI related mortality might actually exist, but in some populations only.

\section{CONCLUSIONS}

In summary, the SMIR database ${ }^{4}$ highlights that ethnic variations in the incidence and, possibly, clinical outcome of acute CAD events probably exist. The causes for such differences, however, are multiple and difficult to fully address, given the complex interplay of contributing factors.

Environmental, nutritional, socioeconomic, cultural, and lifestyle factors certainly play a major role. On the other hand, genetic factors might significantly contribute to ethnic differences variously influencing the susceptibility to risk factors, atherosclerosis, precipitating causes of ACS and, possibly, cardiac arrhythmias. Their actual role, however, remains to be established.

In the meantime, fighting against classical risk factors, which have consistently been associated with CAD events, and intervening timely and appropriately in patients with a suspicion of an ACS remain the major goals for decreasing CAD incidence and mortality in any kind of ethnic groups.

\section{ACKNOWLEDGEMENTS}

I thank Professor Filippo Crea for his critical review of the manuscript and his precious advice. I am also indebted to Dr Greogory A Sgueglia for his valuable assistance in preparing the manuscript.

\section{REFERENCES}

1 National Institutes of Health, National Heart, Lung and Blood Institute. Morbidity and mortality: 2000 chart book on cardiovascular, lung, and blood disease. Bethesda, Maryland: NIH, NHLBI, 2000:34.

2 Tunstall-Pedoe H, Kuulasmaa K, Amouyel P, et al. Myocardial infarction and coronary deaths in the World Health Organization MONICA project: registration procedures, event rates, and case-fatality rates in 38 populations from 21 countries in four continents. Circulation 1994;90:583-612.

3 Gazano JM. Global burden of cardiovascular disease. In: Braunwald E, Zipes DP, Libby P, Eds. Heart disease. Philadelphia: WB Saunders Co, 2001:1-18.

4 Mak KH, Kark JD, Chia KS, et al. Ethnic variations in female vulnerability after an acute coronary event. Heart 2004;90:621-6.

5 Mak KH, Chia KS, Kark JD, et al. Ethnic differences in acute myocardial infarction in Singapore. Eur Heart J 2003;24:151-60.

6 Chaturvedi N. Ethnic differences in cardiovascular disease. Heart 2003;89:681-6. 
7 Anand SS, Yusuf S, Vuksan V, et al. Differences in risk factors, atherosclerosis, and cardiovascular disease between ethnic groups in Canada: the study of health assessment and risk in ethnic groups (SHARE). Lancet 2000;356:279-84.

8 Ranjith N, Pegoraro RJ, Rom L. Haemostatic gene polymorphisms in young indian asian subjects with acute myocardial infarction. Med Sci Monit 2003;9:CR417-21

9 Keil JE, Sutherland SE, Knapp RG, et al. Mortality rates and risk factors for coronary disease in black as compared with white men and women. N Engl J Med 1993:329:73-8.

10 Gillum RF, Mussolino ME, Madans JH. Coronary heart disease incidence and survival in African-American women and men. The NHANES-I epidemiologic follow-up study. Ann Intern Med 1997; 127:111-8.

11 Jha AK, Varosy PD, Kanaya AM, et al. Differences in medical care and disease outcomes among black and white women with heart disease. Circulation 2003;108:1089-94.
12 Pristipino C, Beltrame JF, Finocchiaro ML, et al. Major racial differences in coronary constrictor response between Japanese and Caucasians with recent myocardial infarction. Circulation 2000;101:1102-8.

13 Roig E, Castaner A, Simmons B, et al. In-hospital mortality rates from acute myocardial infarction by race in U.S. hospitals: findings from the national hospital discharge survey. Circulation 1987;76:280-8

14 Stone PH, Thompson B, Anderson HV, et al. Influence of race, sex, and age on management of unstable angina and non-Q-wave myocardial infarction: the TIMI III registry. JAMA 1996;275:1104-12.

15 Tunstall-Pedoe H, Morrison C, Woodward M, et al. Sex differences in myocardial infarction and coronary deaths in the Scottish MONICA population of Glasgow 1985-91: presentation, diagnosis, treatment, and 28.day case fatality of 3991 events in men and women. Circulation 1996:93:1981-92.

16 Andreotti F, Conti E, Lanza GA, et al. Sex, survival bias, and mortality following acute myocardial infarction. Ital Heart J 2003;4:508-10.

\section{IMAGES IN CARDIOLOGY}

\section{Myocardial abscess}

A 53 year old woman underwent emergency laparotomy for small bowel obstruction. Inotropic support was administered via a central venous catheter. Five days postoperatively she became pyrexial, oliguric, and haemodynamically unstable. Multiple areas of peripheral limb infarction were noted. Blood cultures grew Staphylococcus aureus, which was also cultured from her central venous catheter.

Transoesophageal echo (panel A) demonstrated severe mitral and aortic valve regurgitation, a $2 \mathrm{~cm}$ vegetation (V) on the posterior leaflet of her mitral valve, a disorganised aortic valve (A), and echodense areas in the interventricular septum (arrows). She underwent debridement of an aortic root abscess, homograft replacement of her aortic root, and the vegetation was removed from her mitral valve via a left atriotomy. She was weaned from cardiopulmonary bypass, but died on the operating table as a result of uncontrollable haemorrhage.

Postmortem examination showed large myocardial abscesses in the left and right ventricular free wall and interventricular septum (panel B), mitral valve endocarditis, renal abscesses (panel $\mathrm{C}$ ), and splenic and cerebral abscesses.

Multiple myocardial abscesses distant from the valvar apparatus are an uncommon, late, and usually fatal complication of endocarditis. Splenic and renal abscesses are more common. S aureus endocarditis follows a particularly virulent course, and should be treated aggressively, with surgical management considered at an early stage.

J Chikwe

J Barnard

J R Pepper

j.chikwe@medschl.cam.ac.uk
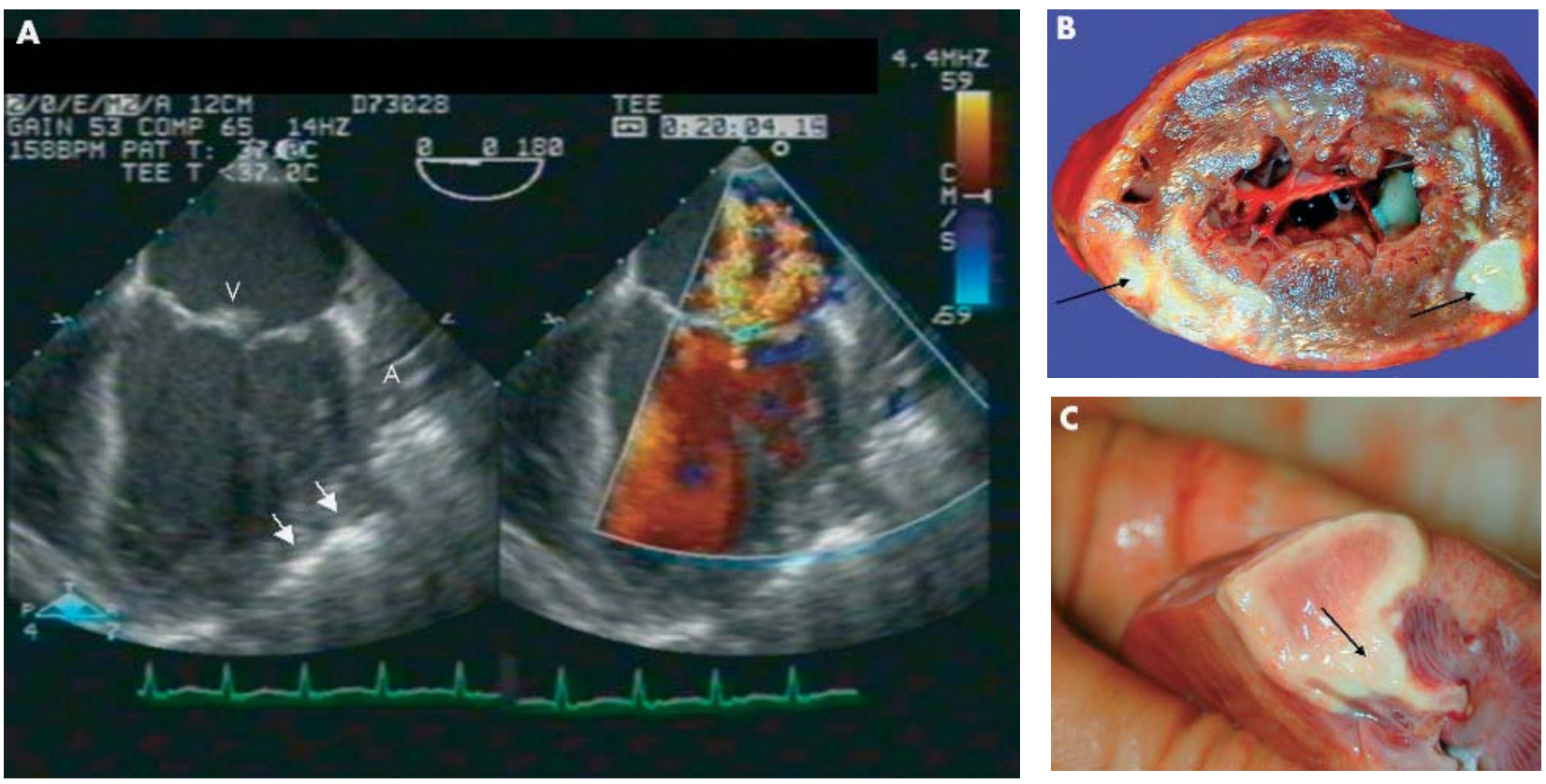